\title{
Group index determination by pulse delay measurements and dispersion study in the zero dispersion region of fused silica
}

\author{
M. Tondusson ${ }^{1,2}$, J. Degert ${ }^{1,2, *}$, F. Couchot ${ }^{3}, \mathrm{X}$.

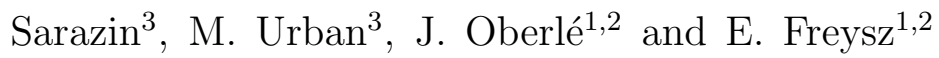 \\ ${ }^{1}$ Univ. Bordeaux, LOMA, UMR 5798, F-33400 Talence, France \\ ${ }^{2}$ CNRS, LOMA, UMR 5798, F-33400 Talence, France \\ ${ }^{3}$ LAL, Univ. Paris-Sud, CNRS/IN2P3, Orsay, France \\ *Corresponding author: j.degert@loma.u-bordeaux1.fr
}

\begin{abstract}
We report on the measurement of the group index of fused silica in the zero dispersion region by pulse delay measurements. Our results differ from the calculated values of the group index from the literature by less than $1 \cdot 10^{-5}$. The dispersion within the $1200-1340 \mathrm{~nm}$ range is also studied by means of auto and cross-correlation measurements. The results are in good agreement with the known dispersion of fused silica.
\end{abstract}

PACS numbers: 


\section{INTRODUCTION}

Fused silica is widely used in optics. The capability to synthesize this dielectric material with very high purity and low hydroxyl group content has revolutionized the development of optical telecom. Thus, fused silica is largely used to produce optical substrates, lenses, or fibers. To give rise to peculiar properties, some dopants are often added. To be able to write permanent gratings in fiber the fused silica can be doped by germanium, tin germanium or germanium boron codopants [1]. The doping with rare earth such as Erbium or Ytterbium makes it possible to realize fiber amplifiers that are largely used in Telecom or to develop high-power laser systems [2]. For all these applications, the dispersion of fused silica, i.e., the dependence of its refractive index $n$ on the wavelength $\lambda$, is a key parameter. For example, when an ultrashort laser pulse passes through a thin piece of fused silica, it gets broadened. The pulse broadening depends mainly on the fused silica thickness and on $n(\lambda)$ and its derivatives at the central wavelength of laser pulse. At $\lambda=1.27 \mu \mathrm{m}$, close to the zero dispersion region of bulk fused silica, the pulse broadening is usually small and negligible. However, higher dispersion orders usually affect the temporal shape of the laser pulse.

So far, the dispersion of fused silica has been measured using different methods. The most commonly used is the minimum deviation method that yields accurate values [3]. However, other techniques using Michelson-like interferometers illuminated by polychromatic light sources have also been used extensively to measure the refractive index, or the group index $n_{g}(\lambda)$, of fused silica with an accuracy of the order of $10^{-4}-10^{-5}$ [4, 5, 6, 7]. Few years ago, Günter et al. proposed a simple method to determine $n$ over a wide wavelength range [8]. It relied on the determination of the group index by measuring the time-retardation, $\Delta t(\lambda)$, that a femtosecond laser pulse undergoes while passing through a fused silica sample. The accuracy of the experience was less than $3 \cdot 10^{-4}$.

In this paper, using the same experimental method as Günter et al., we present very accurate group index mesurements of a fused silica sample in its zero dispersion region. Our experimental values of $n_{g}(\lambda)$, compared to the ones deduced from the Sellmeier dispersion equation proposed by Malitson [3], differ by less than $1 \cdot 10^{-5}$. The increase of our precision is achieved thanks to a dedicated treatment of the experimental data. Indeed, around this spectral range, the temporal shape of the laser pulse envelope is no longer symmetric and the determination of $\Delta t(\lambda)$ requires a lot of care. The parameters that limit the accuracy 
of our measurements are discussed.

\section{THEORETICAL BACKGROUND}

In order to model our experiments, let us consider an ultrashort laser pulse with a carrier frequency $\omega_{\ell}$ (corresponding to a wavelength in vacuum $\lambda_{\ell}$ ) propagating in the $z$-direction, and entering in a transparent medium, with a refractive index $n(\omega)$ and a thickness $L$, in $z=0$. At this point, it is characterized by its complex electric field [9]:

$$
E_{\text {in }}(t)=E(t, z=0)=\frac{1}{2} A_{i}(t) e^{-i\left[\omega_{\ell} t-\varphi_{i}(t)\right]} .
$$

Each spectral component $\omega$ of this pulse is characterized by its complex spectral amplitude, $E_{\text {in }}(\omega)=E(\omega, z=0)$, null for negative frequencies, and defined by [9, 10]:

$$
\begin{aligned}
E_{\text {in }}(\omega) & =a(\omega) e^{i \phi_{i}(\omega)}, \\
& =\int_{-\infty}^{+\infty} E_{i n}(t) e^{i \omega t} d t
\end{aligned}
$$

At the exit of the medium, owing to dispersion, the complex spectral amplitude of the pulse, $E_{\text {out }}(\omega)=E(\omega, z=L)$, is given by $[9]$ :

$$
\begin{aligned}
E_{\text {out }}(\omega) & =a(\omega) e^{i \phi_{o}(\omega)}, \\
& =E_{\text {in }}(\omega) e^{i \phi(\omega)},
\end{aligned}
$$

with $\phi(\omega)=\phi_{o}(\omega)-\phi_{i}(\omega)=k(\omega) L=\omega n(\omega) L / c, c$ being the velocity of light in vacuum. Note that, experimentally, one has access to the spectral intensity, given by $[9$ :

$$
S(\omega)=\frac{\varepsilon_{0} c n\left(\omega_{\ell}\right)}{\pi}\left|E_{\text {out }}(\omega)\right|^{2}=\frac{\varepsilon_{0} c n\left(\omega_{\ell}\right)}{\pi}\left|E_{\text {in }}(\omega)\right|^{2} .
$$

Moreover, since $\omega_{\ell}$ is far from the resonance frequencies $\omega_{k}$ of the medium and the full width at half maximum (FWHM) $\Delta \omega_{\ell}$ of $S(\omega)$ is much smaller than $\omega_{\ell}, \phi(\omega)$ can be expanded in the neighborhood of $\omega_{\ell}$ :

$$
\phi(\omega)=\phi_{\ell}+\sum_{m} \frac{1}{m !} \phi_{\ell}^{(m)}\left(\omega-\omega_{\ell}\right)^{m},
$$

with $\phi_{\ell}=\phi\left(\omega_{\ell}\right)=k\left(\omega_{\ell}\right) L$ and $\phi_{\ell}^{(m)}=\left(d^{m} \phi / d \omega^{m}\right)_{\omega_{\ell}}$. Generally, only the first few terms of the Taylor expansion are taken into account, the corresponding $\phi_{\ell}^{(m)}$ coefficients being calculated thanks to the knowledge of $n(\omega)$ or $n(\lambda)$. In the visible and near-infrared spectral 
ranges, $n$ depends on the wavelength in vacuum $\lambda$ according to the Sellmeier dispersion equation [10, 11]:

$$
n^{2}(\lambda)-1=\sum_{k} \frac{B_{k} \lambda^{2}}{\lambda^{2}-\lambda_{k}^{2}},
$$

where the $\lambda_{k}$ 's are the resonance wavelengths of the medium, lying in the ultraviolet.

Usually, the ultrashort pulse exiting from the medium, with a complex electric field

$$
E_{\text {out }}(t)=E(t, z=L)=\frac{1}{2} A_{o}(t) e^{-i\left[\omega_{\ell} t-\varphi_{o}(t)\right]},
$$

is characterized by its bandwidth $\Delta \omega_{\ell}$ and its duration $\tau_{p}$ defined as the FWHM of the intensity

$$
I_{o}(t)=\frac{1}{2} \varepsilon_{0} c n\left(\omega_{\ell}\right) A_{o}^{2}(t)
$$

For Fourier limited (FL) pulses, such that $\phi_{i}(\omega)=\phi(\omega)=0 \forall \omega$, we have $\tau_{p} \Delta \omega_{\ell}=2 \pi K$, where $K$ is a constant on the order of $1 / 2$, depending on the pulse shape. However, for a propagation through an optically dense medium, the reshaping of the pulse at the exit of the medium may be such that it is no longer relevant to talk about temporal and spectral FWHM, particularly if the pulse shape exhibits pre- or postsubpulses that lengthen considerably the pulse and are not accounted for by the temporal FWHM $\tau_{p}$. As we faced such a situation in our experiment (see section IV), we found more convenient to define the characteristics of the pulse, in both the spectral and temporal domains, in terms of standard deviation (noted $\sigma$ hereafter) calculated with $I(t)$ or $S(\omega)$ as a probability density. To this end, one introduces the moment of order $m$ for the time $t$ and the frequency $\omega$ as defined by $[9]$ :

$$
\begin{aligned}
\left\langle t^{m}\right\rangle & =\int_{-\infty}^{+\infty} t^{m} I(t) d t / \int_{-\infty}^{+\infty} I(t) d t \\
\left\langle\omega^{m}\right\rangle & =\int_{0}^{+\infty} \omega^{m} S(\omega) d \omega / \int_{0}^{+\infty} S(\omega) d \omega .
\end{aligned}
$$

The pulse width, $\sigma_{t}$, and the spectral width, $\sigma_{\omega}$, are then defined as

$$
\begin{aligned}
\sigma_{t} & =\sqrt{\left\langle(t-\langle t\rangle)^{2}\right\rangle}=\sqrt{\left\langle t^{2}\right\rangle-\langle t\rangle^{2}} \\
\sigma_{\omega} & =\sqrt{\left\langle(\omega-\langle\omega\rangle)^{2}\right\rangle}=\sqrt{\left\langle\omega^{2}\right\rangle-\langle\omega\rangle^{2}}
\end{aligned}
$$

with [9]:

$$
\sigma_{t} \times \sigma_{\omega} \geq 1 / 2
$$


the equality holding only for a Fourier limited Gaussian-shaped-pulse.

Furthermore, this description in terms of standard deviation makes it possible to connect the pulsewidth at the exit of the medium to its initial value, this at all the orders of dispersion, something impossible to do with a FWHM description. To show this, let us note $\sigma_{o}$ the pulsewidth at the exit of the transparent medium (defined by Eq. (13) with $I(t)=I_{o}(t)$ ), and $\sigma_{i}$ its width at the entrance (Eq. (13) with $I(t)=I_{i}(t)$ ), assuming the input pulse is Fourier limited $\left(\phi_{i}(\omega)=0\right)$. Using the results demonstrated in Ref. [9] $\S 1.1 .4$, one obtains:

$$
\sigma_{o}^{2}=\sigma_{i}^{2}+\left\langle T^{2}(\omega)\right\rangle-\langle T(\omega)\rangle^{2}
$$

where $T(\omega)=d \phi(\omega) / d \omega=L / v_{g}(\omega)$ is the group delay at frequency $\omega$, that is the time it takes to the spectral component $\omega$ to propagate through the medium at the group velocity $v_{g}(\omega)=d \omega / d k=c / n_{g}(\omega)$. By introducing the standard deviation

$$
\sigma_{T}=\sqrt{\left\langle T^{2}(\omega)\right\rangle-\langle T(\omega)\rangle^{2}}
$$

of the group delay, Eq. (16) takes the simple form:

$$
\sigma_{o}^{2}=\sigma_{i}^{2}+\sigma_{T}^{2}
$$

Eq. (18) clearly highlights the role the dispersion of the group delay, i.e., the group velocity dispersion (GVD), plays in the stretching of an ultrashort pulse in a transparent medium.

In theory, Eqs. (13), (14), (17) and (18) can be used for the processing/modeling of the data obtained in the next sections. However, in our experiments, we did not measure the pulses intensity $I(t)$, so that we cannot define $\left\langle t^{m}\right\rangle$ and $\sigma_{t}$ as in Eqs. (11) and (13). On the other hand, we measured the intensity autocorrelation, $A_{i}(\tau)$, of the pulse at the entrance of the medium:

$$
A_{i}(\tau)=\int_{-\infty}^{+\infty} I_{i}(t) I_{i}(t-\tau) d t
$$

and its intensity cross-correlation, $C_{o}(\tau)$, at the exit of the medium:

$$
C_{o}(\tau)=\int_{-\infty}^{+\infty} I_{o}(t) I_{i}(t-\tau) d t
$$

From $C_{o}(\tau)$, one can determine the pulsewidth at the exit of the medium, $\sigma_{o}$, knowing its width, $\sigma_{i}$, at the entrance. Indeed, let us introduce the output cross-correlation width, $\sigma_{c}$, defined by:

$$
\sigma_{c}=\sqrt{\left\langle\tau^{2}\right\rangle_{c}-\langle\tau\rangle_{c}^{2}}, \text { with }\left\langle\tau^{m}\right\rangle_{c}=\frac{\int_{-\infty}^{+\infty} \tau^{m} C_{o}(\tau) d \tau}{\int_{-\infty}^{+\infty} C_{o}(\tau) d \tau}
$$


$\sigma_{o}, \sigma_{i}$ and $\sigma_{c}$ are then linked by the equation:

$$
\sigma_{c}^{2}=\sigma_{o}^{2}+\sigma_{i}^{2} \Longrightarrow \sigma_{o}=\sqrt{\sigma_{c}^{2}-\sigma_{i}^{2}}
$$

For data processing, we will use Eqs. (21) and (22) with the following definition for $\sigma_{i}$ :

$$
\sigma_{i}=\frac{1}{\sqrt{2}} \sqrt{\left\langle\tau^{2}\right\rangle_{i}-\langle\tau\rangle_{i}^{2}}, \text { with }\left\langle\tau^{m}\right\rangle_{i}=\frac{\int_{-\infty}^{+\infty} \tau^{m} A_{i}(\tau) d \tau}{\int_{-\infty}^{+\infty} A_{i}(\tau) d \tau}
$$

in agreement with Eqs. (21) and (22) applied to an autocorrelation.

\section{EXPERIMENTAL SETUP}

We have used two experimental setups: one for measuring the group index $n_{g}$ of fused silica in its zero dispersion region, and an other one to study its dispersion in the same spectral range. Both are using the same laser system: a modelocked Ti:Sapphire oscillator (Chameleon, Coherent) providing ultrashort pulses of duration $\sim 140 \mathrm{fs}$ at $830 \mathrm{~nm}$ with a $80 \mathrm{MHz}$ repetition rate is used to pump an OPO (Chameleon-OPO, Coherent) tunable in the $1200-1350 \mathrm{~nm}$ range, delivering pulses of duration $\tau_{p} \sim 200 \mathrm{fs}$.

The first setup is shown on Fig. 1. The output of the OPO is split in two parts, one propagating through a removable fused silica rod of length $L=20 \mathrm{~cm}$, the other one propagating in air. These two beams are correlated in a $10 \mu$ m-thick type I BBO crystal. The autocorrelation of the pulses delivered by the OPO is measured by an autocorrelator (APE, PulseCheck Autocorrelator version 15). The travel of the translation stage is long enough to make it possible to measure the cross correlation, $C_{o}(\tau)$, with the rod, and the cross correlation, $C_{o}^{(\text {air })}(\tau)$, without it. The spectral intensity $S(\omega)$ at the output of the OPO is also recorded by a spectrum analyzer (Anritsu MS9710C).

The second setup is shown on Fig. 2, Like in the first one, two pulses, one propagating in fused silica, the other one propagating in air, are correlated in a $100 \mu$ m-thick type I $\mathrm{BBO}$ crystal. However, since the wavelength of the pulses is lying within the zero dispersion region of fused silica, the number of crossings through the silica rod is increased in order to get an appreciable stretching of the pulses. To this end, the rod is inserted within a Herriot Cell [12] constructed with a plane gold mirror and a concave gold mirror (radius of curvature $R_{c}=1.80 \mathrm{~m}$ ) separated by a distance of $25 \mathrm{~cm}$. Thanks to this setup, one of the two pulses performs 10 round trips within the rod, i.e., it passes through $L=4 \mathrm{~m}$ of fused 
silica. For this experiment, we also measured the autocorrelation and the spectral intensity at the output of the OPO.

\section{RESULTS AND DISCUSSION}

Fig. 3 displays the measured group index of fused silica with its standard deviation (blue squares) for wavelengths ranging from 1200 to $1350 \mathrm{~nm}$. It has been determined in the following way: from the cross correlations with the rod and without it, we have calculated the time interval $t_{\text {silica }}(L, \lambda)$ (resp. $\left.t_{\text {air }}(L, \lambda)\right)$ required for the pulse to propagate through a length $L=20 \mathrm{~cm}$ of fused silica (resp. air), defined as:

$$
\begin{aligned}
t_{\text {silica }}(L, \lambda) & =\frac{\int_{-\infty}^{+\infty} \tau C_{o}(\tau) d \tau}{\int_{-\infty}^{+\infty} C_{o}(\tau) d \tau}, \\
t_{\text {air }}(L, \lambda) & =\frac{\int_{-\infty}^{+\infty} \tau C_{o}^{(\text {air })}(\tau) d \tau}{\int_{-\infty}^{+\infty} C_{o}^{(\text {air })}(\tau) d \tau} .
\end{aligned}
$$

The retardation $\Delta t(L, \lambda)$ between the two pulses propagating respectively in silica and air is then given by: $\Delta t(L, \lambda)=t_{\text {silica }}(L, \lambda)-t_{\text {air }}(L, \lambda)$. Assuming that $t_{\text {silica }} \equiv\langle t\rangle_{o}$ and $t_{\text {air }} \equiv\langle t\rangle_{o}^{(\text {air })}$, where $\langle t\rangle_{o}$ (resp. $\langle t\rangle_{o}^{(\text {air })}$ ) is the first-order moment of the time $t$ for a pulse propagating through silica (resp. air), and knowing that [9]:

$$
\langle t\rangle=\left\langle\frac{d \phi_{o}}{d \omega}\right\rangle=\frac{L}{c}\left\langle n_{g}(\lambda)\right\rangle
$$

we have for the group index, $n_{g}$, of fused silica:

$$
\left\langle n_{g}(\lambda)\right\rangle=\left\langle n_{g, \text { air }}(\lambda)\right\rangle+\frac{c \Delta t}{L}
$$

Since $\Delta \omega_{\ell} / \omega_{\ell} \ll 1,\left\langle n_{g}(\lambda)\right\rangle \equiv n_{g}\left(\lambda_{\ell}\right)$ and $\left\langle n_{g, \text { air }}(\lambda)\right\rangle \equiv n_{g, \text { air }}\left(\lambda_{\ell}\right)$ to a precision lower by one order of magnitude than the standard deviation of the data as shown by numerical computation, $\lambda_{\ell}$ being defined as:

$$
\lambda_{\ell}=\langle\lambda\rangle=\int_{0}^{+\infty} \lambda S(\lambda) d \lambda / \int_{0}^{+\infty} S(\lambda) d \lambda .
$$

Note that this latter definition of $\lambda_{\ell}$ overcomes the difficulties in defining precisely the center wavelength of the pulse, especially for broad, asymmetric spectra, and offers an unambiguous determination of the wavelength at which the group index is measured. Thus, Eq. (27) leads to:

$$
n_{g}\left(\lambda_{\ell}\right)=n_{g, \text { air }}\left(\lambda_{\ell}\right)+\frac{c \Delta t}{L}
$$


the dispersion of air, and consequently $n_{g \text {,air }}$, being known with a high precision from the literature [13]. The results given by Eq. (29) are compared to the group indices calculated analytically from the Sellmeier equation given in Ref. [3] using the following equation [14]:

$$
n_{g}\left(\lambda_{\ell}\right)=n\left(\lambda_{\ell}\right)-\lambda_{\ell}\left(\frac{d n}{d \lambda}\right)_{\lambda_{\ell}} .
$$

As shown by Fig. 3, our results differ from the calculated values of the group index by less than $\pm 1 \cdot 10^{-5}$ for all measurements, corresponding to an improvement by one order of magnitude compared to the results of Ref. [8] in the 1200-1350 nm spectral range.

Let us now discuss some points concerning the possible sources of uncertainty in the measured values of $n_{g}$. One source is the variation of the sample temperature. Indeed, the data shown on Fig. 3 correspond to an average of several days of experience. During these days, the sample temperature $\theta$ varied from $20.3^{\circ} \mathrm{C}$ to $20.8^{\circ} \mathrm{C}$. According to [3], it corresponds to a change, $(\delta n)_{\theta}$, of the refractive index of fused silica equal to $\sim 5 \cdot 10^{-6}$. Note that this temperature variation also leads to a change of the refractive index of air of $\sim 6 \cdot 10^{-7}[15]$. Owing to its value, this latter can be discarded as a possible source of error in our experiment. Another source of uncertainty is the accuracy concerning the knowledge of $L$. Thanks to flatness and length measurements of our rod, $L$ is known with an uncertainty $\delta L=1 \mu \mathrm{m}$, leading to an uncertainty on $n_{g}:\left(\delta n_{g}\right)_{L}=c \Delta t \delta L / L^{2} \sim 2 \cdot 10^{-6}$, since $\Delta t \sim 300 \mathrm{ps}$ for $20 \mathrm{~cm}$ of fused silica. Note also that, in our experience, the time step of the auto and cross correlations was $3.33 \mathrm{fs}$, meaning that $\Delta t$ is measured with an uncertainty $\delta(\Delta t)=3.33 \mathrm{fs}$. This limit also introduces an uncertainty on the determination of $n_{g}$ which is: $\left(\delta n_{g}\right)_{\Delta t}=c \delta(\Delta t) / L \sim 6 \cdot 10^{-6}$. All these sources of uncertainty lead to an overall uncertainty, $\delta n_{g}$, given by:

$$
\delta n_{g}=\sqrt{(\delta n)_{\theta}^{2}+\left(\delta n_{g}\right)_{L}^{2}+\left(\delta n_{g}\right)_{\Delta t}^{2}} \sim 8 \cdot 10^{-6},
$$

in agreement with the experiment. Finally, let us notice again that the approximation $\left\langle n_{g}(\lambda)\right\rangle \equiv n_{g}(\langle\lambda\rangle)$ introduces an overestimation of the group index of about $1 \cdot 10^{-6}$. To conclude this paragraph, we must remark that we obtained very accurate values of the group index thanks to the use of a very thick sample $(L=20 \mathrm{~cm})$, leading to a relative uncertainty, $\delta L / L$, on $L$ of $5 \cdot 10^{-6}$. Working with thinner samples would lead inevitably to a loss of precision, since $\delta L / L$ would increase. For example, in Ref. [8], a (3.040 \pm 0.001$) \mathrm{mm}$ thick plate of fused silica was used (i.e., $\delta L / L=3.3 \cdot 10^{-4}$ ). With this sample, the uncertainties 
$\left(\delta n_{g}\right)_{L}$ and $\left(\delta n_{g}\right)_{\Delta t}$ are respectively about $1.5 \cdot 10^{-4}$ and $3.3 \cdot 10^{-4}$, giving an overall uncertainty $\delta n_{g} \sim 4 \cdot 10^{-4}$, in agreement with the results of [8].

Fig. 4 presents some typical results obtained during the study of the dispersion of fused silica. Here, the spectral intensity is centered at the mean wavelength $\lambda_{\ell}=1200 \mathrm{~nm}$. Fig. 4b displays the autocorrelation, $A_{i}(\tau)$, of the corresponding pulses at the entrance of the Herriot cell, which is compared to the autocorrelation signal, $A_{\mathrm{FL}}(\tau)$, of Fourier limited pulses. This latter is calculated from the experimental spectral intensity thanks to the relation between $S(\omega)$ and the intensity of a Fourier limited pulse:

$$
I_{\mathrm{FL}}(t) \propto\left|\mathcal{F}^{-1}[\sqrt{S(\omega)}]\right|^{2}
$$

where $\mathcal{F}$ denotes the complex Fourier transform. As can be seen, the pulses are not Fourier limited at the entrance of the cell. This must be taken into account in order to model correctly the dispersion introduced by the whole experimental setup. Note that the intensity $I_{\mathrm{FL}}(t)$ also makes it possible to calculate the temporal width, $\sigma_{\mathrm{FL}}$, of the Fourier limited pulses. Concerning the pulses at the exit of the Herriot cell, the cross correlation of Fig. 4c clearly shows that their temporal shape is no longer symmetric. This phenomenon, resulting from the presence of significant high orders of dispersion, confirms the need to go beyond a simple description of the pulses at the output of a dispersive medium in terms of FWHM as already stressed in section II.

The whole results concerning the study of the dispersion of fused silica in the 1200$1340 \mathrm{~nm}$ range are summed up in Fig. 5. It shows the pulsewidths before (circles) and after (up triangles) propagation through $4 \mathrm{~m}$ of silica. In agreement with Fig. 4, the pulses are not Fourier limited at the entrance of the rod since, for some wavelengths, we have $\sigma_{o}<\sigma_{i}$. One can also notice that $\sigma_{o} \simeq \sigma_{i}$ for $\lambda_{\ell} \sim 1270 \mathrm{~nm}$ and $\sim 1305 \mathrm{~nm}$, the former wavelength agreeing with the existence of the so-called zero material dispersion wavelength around $1270 \mathrm{~nm}$ in fused silica [14]. At this latter wavelength, the pulse is supposed to undergo a very weak dispersion, and its temporal width must remain almost unchanged. We have also calculated from the Fourier transform of $\sqrt{S(\omega)}$ the Fourier limited pulsewidths $\sigma_{\mathrm{FL}}$ (black squares). As expected, we observe that $\sigma_{o} \geq \sigma_{\mathrm{FL}}$, the equality being reached for $\lambda_{\ell} \sim 1290 \mathrm{~nm}$. At this wavelength, the frequency chirp of the input pulse is compensated by the dispersion of the medium. Therefore, the pulse retrieves its Fourier limited pulsewidth. Indeed, assuming that the pulses delivered by the OPO have a quadratic phase modulation 
in the frequency domain, it leads to an initial group delay:

$$
T_{i}(\omega)=\frac{d \phi_{i}}{d \omega}=\phi_{i \ell}^{(2)}\left(\omega-\omega_{\ell}\right)
$$

with $\phi_{i \ell}^{(2)}=\sqrt{\sigma_{i}^{2}-\sigma_{\mathrm{FL}}^{2}} / \sigma_{\omega}$. At $1290 \mathrm{~nm}, \phi_{i \ell}^{(2)} \simeq 7100 \mathrm{fs}^{2}$ and the dispersion in fused silica is dominated by the GVD, i.e., is quadratic, with $\phi_{\ell}^{(2)} \simeq-6000 \mathrm{fs}^{2}$. These two contributions lead to a residual quadratic phase at the exit of the medium corresponding to an output pulsewidth $\sigma_{o}$ differing of $\sigma_{\mathrm{FL}}$ by less than $1 \mathrm{fs}$. Now, for wavelengths above $1290 \mathrm{~nm}$, more dispersion is added to the Fourier limited pulse. Hence, at $\sim 1305 \mathrm{~nm}$, the pulse width is equal again to the pulse width at the entrance of the rod, but its frequency chirp is now opposite in sign.

Finally, Fig. 5 displays the calculated pulsewidth at the exit of the Herriot cell (down triangles). For this calculation, we have used Eq. (18): the group delay, $T(\omega)$, of fused silica is computed from Eq. (7) limited to the fifth-order derivative, taking for the refractive index $n(\lambda)$ the one given in Ref. [3]. The computed pulsewidth at the output of the cell is then given by:

$$
\sigma_{o}=\sqrt{\sigma_{\mathrm{FL}}^{2}+\sigma_{T_{o}}^{2}}
$$

with $T_{o}(\omega)=T_{i}(\omega)+T(\omega)$. In this latter equation, $\sigma_{T_{o}}$ is calculated by means of the spectral intensity recorded in the course of the experience. Given the fact that we do not fully characterize the electric field (amplitude and phase) of the pulses, especially at the output the OPO, the agreement between the experimental and the calculated output pulsewidths is rather good other the entire spectral range explored here.

\section{CONCLUSION}

In conclusion, we have proposed an experimental method that makes it possible to determine accurately the group index $n_{g}(\lambda)$ of a transparent sample near the zero dispersion region. This techniques has been applied to the determination of $n_{g}(\lambda)$ for fused silica. Our data are found to very well agree with the values one can derive according to the Sellmeier equation proposed by Malitson [3]. The accuracy of our measurement is $\sim 1 \cdot 10^{-5}$ thanks to the thick sample we used and our data analysis procedure. We have also measured the dispersion of femtosecond pulses within the 1200-1340 nm range. This ensemble of data is important for the description of pulse propagation and nonlinear optical effects in fibers 
such as white light continuum generation in optical fiber near the zero dispersion wavelength. This method can be easily applied to characterization of other transparent linear and nonlinear optical materials close to the zero dispersion region.

\section{Acknowledgments:}

The Conseil Régional d'Aquitaine is acknowledged for financial support.

[1] R. Kashyap, Fiber Bragg Gratings (Academic Press, 1999).

[2] G. P. Agrawal, Nonlinear Fiber Optics (Academic Press, 2001).

[3] I. H. Malitson, "Interspecimen Comparison of the Refractive Index of Fused Silica," J. Opt. Soc. Am. 55, 1205-1209 (1965).

[4] Z. Bor, K. Osvay, B. Rácz, G. Szabó, "Group refractive index measurement by Michelson interferometer," Opt. Comm. 78, 109-112 (1990).

[5] V. N. Kumar, D. N. Rao, "Using interference in the frequency domain for precise determination of thickness and refractive indices of normal dispersive materials," J. Opt. Soc. Am. B. 12, 1559-1563 (1995).

[6] S. Diddams, J.-C. Diels, "Dispersion measurements with white-light interferometry," J. Opt. Soc. Am. B. 13, 1120-1129 (1996).

[7] P. Hlubina, "Group velocity dispersion in fused-silica sample measured using white-light interferometry with the equalization wavelength determination," Optik 113, 149-152 (2002).

[8] A. Schneider, F. D. J. Brunner, P. Günter, "Determination of the refractive index over a wide wavelength range through time-delay measurements of femtosecond pulses," Opt. Comm. 275, 354-358 (2007).

[9] J.-C. Diels, W. Rudolph, Ultrashort Laser Pulse Phenomena (Academic Press, 2006).

[10] M. Born, E. Wolf, Principles of Optics (Cambridge University Press, 1999).

[11] P. Atkins, R. Friedman, Molecular Quantum Mechanics (Oxford University Press, 2005).

[12] D. Herriot, H. Kogelnik, R. Kompfner, "Off-axis paths in spherical mirror interferometers," Appl. Opt. 3, 523-526 (1964).

[13] E. R. Peck, K. Reeder, "Dispersion of air," J. Opt. Soc. Am. 62, 958-962 (1972). 
[14] A. Ghatak, K. Thyagarajan, Introduction to Fiber Optics (Cambridge University Press, 1998).

[15] B. Edlén, "The Refractive Index of Air," Metrologia 2, 71-80 (1966). 


\section{Figure captions:}

Fig. 1 Experimental setup for the measurement of the group index $n_{g}$. BS, beamsplitter.

Fig. 2 Experimental setup for the study of the dispersion of fused silica in the nearinfrared. BS, beamsplitter.

Fig. 3 Group index of fused silica. The blue squares correspond to the measured group index, whereas the solid line corresponds to the group index calculated from the Sellmeier equation given in [3].

Fig. 4 Dispersion of fused silica at $\lambda_{\ell}=1200 \mathrm{~nm}$ : (a) spectral intensity; (b) autocorrelation signal for a FL pulse $\left(A_{\mathrm{FL}}(\tau)\right)$ and at the entrance of the Herriot cell $\left(A_{i}(\tau)\right) ;(\mathrm{c})$ cross-correlation signal, $C_{o}(\tau)$, at the output of the Herriot cell.

Fig. 5 Dispersion of fused silica. $\sigma_{\mathrm{FL}}$ is the pulsewidth of the FL pulses, and $\sigma_{i}$ (resp. $\left.\sigma_{o}\right)$ the pulsewidth at the entrance (resp. exit) of the Herriot cell. 
Figures:

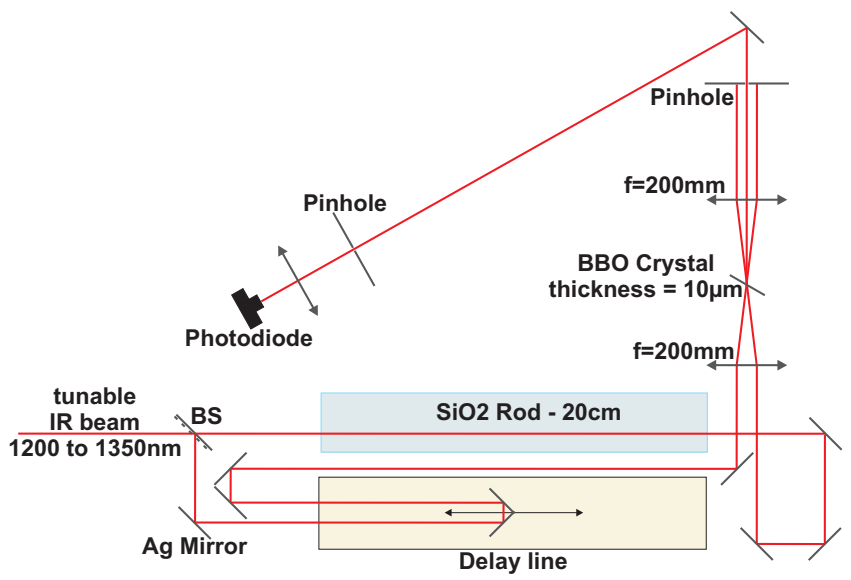

FIG. 1:

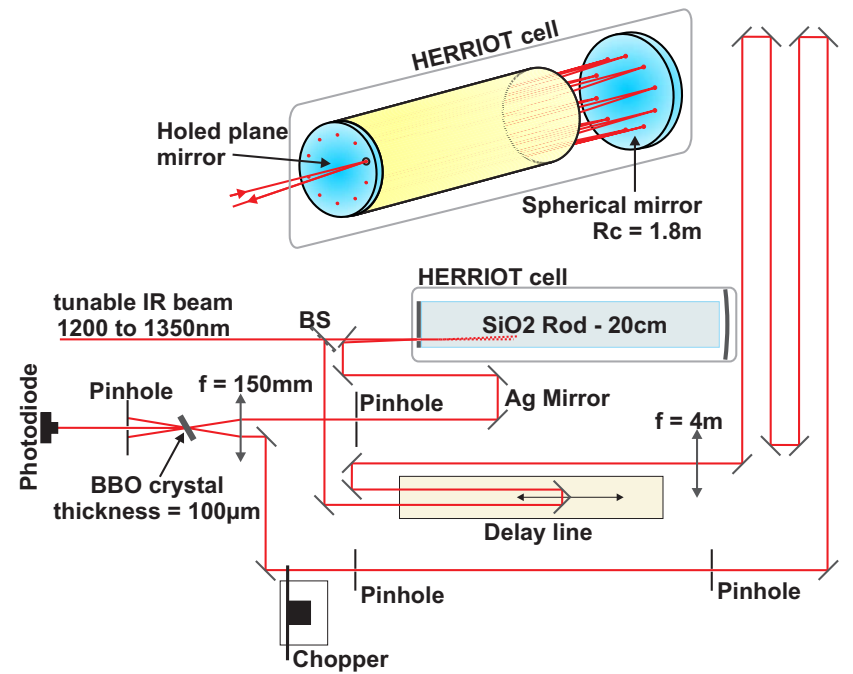

FIG. 2: 


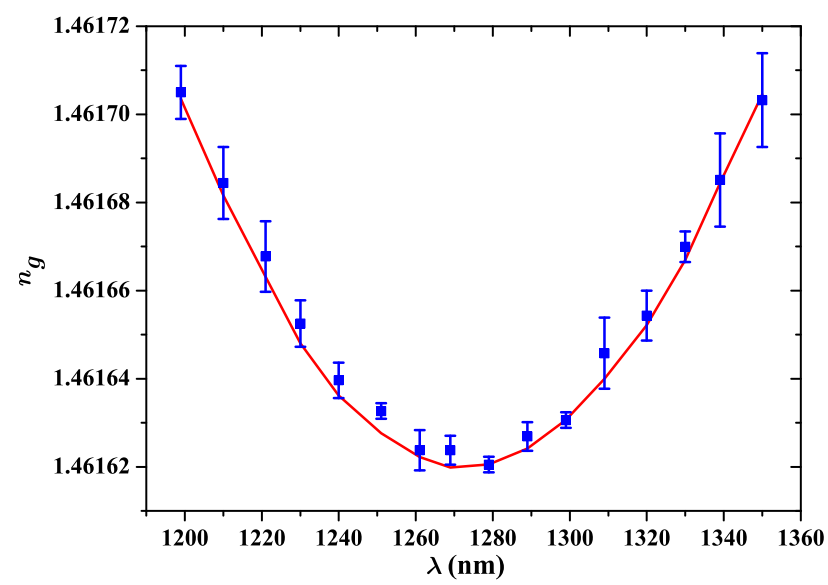

FIG. 3:
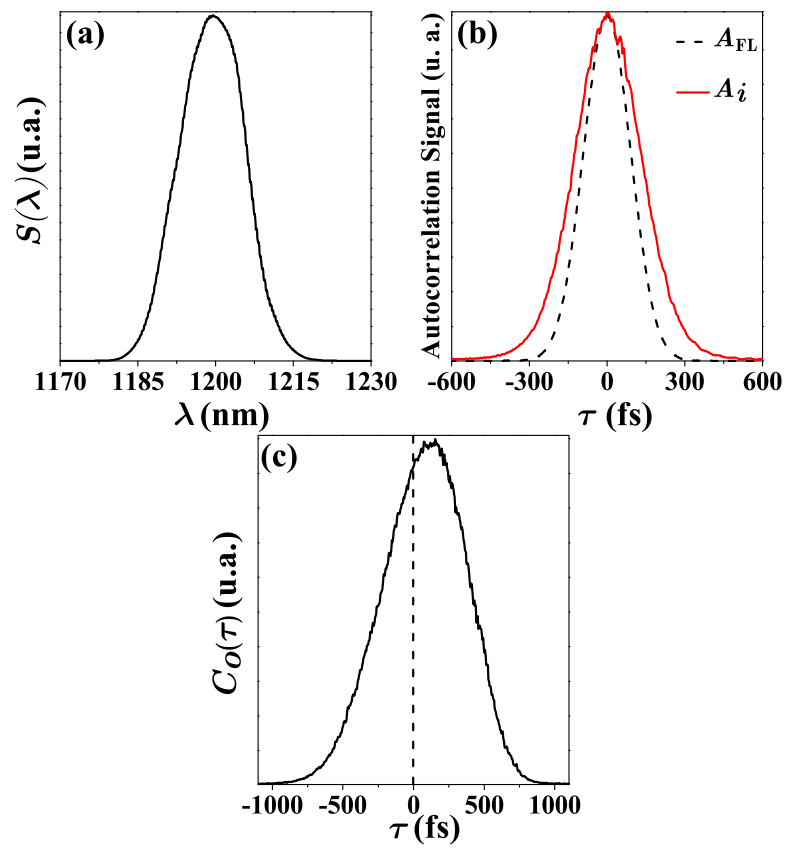

FIG. 4: 


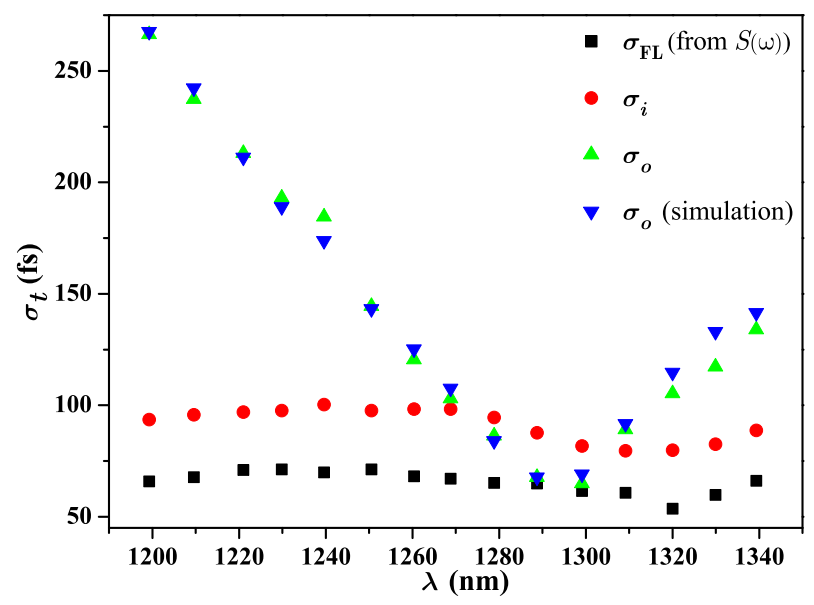

FIG. 5: 